\title{
Correction to: Conclusion
}

\section{Correction to:}

\section{Chapter 12 in: G. O'Shea Brown, Healing Complex}

Posttraumatic Stress Disorder, Essential Clinical Social Work Series, https://doi.org/10.1007/978-3-030-61416-4_12

The original version of the chapter was inadvertently published with an incorrect author's name and affiliation tagged as "Roman Shvydkoy, Math, Stats, \& Comp Sci, University of Illinois at Chicago, Chicago, USA." This has now been corrected as "Gillian O'Shea Brown, New York University, New York, USA." 